\title{
Targeted neonatal echocardiography (TNE) consult service in a large tertiary perinatal center in Canada
}

\author{
Ismina Papadhima ${ }^{1} \cdot$ Deepak Louis $^{1} \cdot$ Jyotsna Purna ${ }^{2} \cdot$ Poorva Deshpande $^{1,2} \cdot$ Yenge Diambomba $^{1,2} \cdot$ Shoo Lee $^{1,2,3}$. \\ Prakesh Shah ${ }^{1,2,3} \cdot$ Dany Weisz $^{4} \cdot$ Afif El-Khuffash ${ }^{5} \cdot$ Patrick J. McNamara $^{2,6} \cdot$ Luc Mertens $^{2,7} \cdot$ Amish Jain $^{1,2,3}$
}

Received: 31 January 2018 / Revised: 2 April 2018 / Accepted: 18 April 2018 / Published online: 22 May 2018

(c) Nature America, Inc., part of Springer Nature 2018

\begin{abstract}
Objective To describe the utilization and study the factors associated with the impact on clinical management of a new TNE consultation service in a perinatal center.

Methods This retrospective cohort study included all neonates who underwent TNE consultation at the neonatal unit of Mount Sinai Hospital in Toronto, Canada (November 2011 and July 2015). The consults that had "impact" were defined as those that led to a TNE suggested change in the clinical management within $6 \mathrm{~h}$ of its recommendation. Logistic regression analysis was performed to identify factors associated with a change in clinical management following the consultation. Results A total of 553 consults were performed for 268 infants (gestational age: $27 \pm 4$ weeks and age at initial consult: 16 $(5,34)$ days). Patent ductus arteriosus (PDA, 61\%), suspected pulmonary hypertension (PH, 27\%), and systemic hypotension ( $\mathrm{SH}, 9 \%)$ were the common indications. The average consultations increased from 9 in 2012-2013 to 20 per month in 2014-2015. Forty eight percent of consults had an impact on clinical management (PDA scans: 38\%, PH: 58\%, and SH: $81 \%, p<0.01$ between all). Male gender (adjusted odds ratio (95\% confidence interval): $1.9(1.0,3.5) ; p=0.04)$, mechanical ventilation $(2.43(1.2,4.9) ; p=0.01)$, and scans for PH $(7.1(2.2,23.2) ; p<0.01)$ and SH $(2.6(1.1,6.5) ; p=$ 0.03 ) were independently associated with the impact on clinical management. TNE consults identified all incidental cases of major structural defects $(n=4)$, and six out of ten minor diagnoses.

Conclusions TNE consult service demonstrated an increasing utilization and a significant impact on clinical management over time especially for non-PDA indications and in situations of high-illness severity. Although, all major cardiac defects were identified, some minor congenital defects were missed by TNEs.
\end{abstract}

\section{Introduction}

Targeted neonatal echocardiography (TNE) refers to pointof-care functional echocardiogram usually performed by neonatologists in the neonatal intensive care unit (NICU) [1].

These authors contributed equally: Ismina Papadhima, Deepak Louis

Amish Jain

amish.jain@sinaihealthsystem.ca

1 Department of Pediatrics, Mount Sinai Hospital, Toronto, ON, Canada

2 Department of Pediatrics, University of Toronto, Toronto, ON, Canada

3 Lunenfeld Tanenbaum Research Institute, Mount Sinai Hospital, Toronto, ON, Canada
The primary goals for performing TNEs include assessment of ventricular function, measurement of variables related to pulmonary and systemic hemodynamics and to evaluate the presence and significance of intra- and extra-cardiac shunts [2]. TNE is also used in the assessment of catheter tip position for central venous catheters, for diagnosing pericardial effusions and in the evaluation of infants with unexpected cardiovascular collapse [3, 4].

4 Department of Newborn and Developmental Pediatrics, Sunnybrook Hospital, Toronto, ON, Canada

5 Department of Pediatrics, Royal College of Surgeons in Ireland, Dublin, Ireland

6 Division of Neonatology, Hospital for Sick Children, Toronto, ON, Canada

7 Division of Pediatric Cardiology, Hospital for Sick Children, Toronto, ON, Canada 
There are several potential advantages of having an inhouse TNE program in the NICU. These include the provision of immediate and possibly off-hour hemodynamic assessment in case of emergencies. In addition, the cardiovascular physiology of a sick neonate may evolve such that frequent longitudinal evaluations may be needed to understand the pathophysiology, guide therapeutic decisions, and assess response to treatment [2]. However, urgent assessments are often not feasible in tertiary NICUs located in perinatal centers without in-house pediatric sub-speciality services. Even in centers with pediatric cardiology services, frequent sequential functional scans can rarely be accommodated. Encouraged by the establishment of expert consensus on standards and guidelines, endorsed by the American Society of Echocardiography (ASE), an increasing number of TNE programs are now being established in NICUs across North America; however, our understanding of its optimal use and impact is still evolving [5-9].

Mount Sinai Hospital (MSH) at the University of Toronto is one of the first perinatal units in Canada to establish a comprehensive in-house TNE consult service, developed in collaboration with the regional pediatric cardiology echocardiography laboratory, and in compliance with the ASE guidelines. In this current study, our aim was to describe the evolution of the clinical utilization of our TNE program over the first 4 years of its introduction, audit its compliance with the published guidelines and study factors associated with its impact on clinical management. We hypothesized that TNE would have an increasing utilization in the NICU over the study period and that it would make a significant impact on clinical management.

\section{Methods}

\section{Design and study settings}

This retrospective cohort study included all neonates who were admitted to the NICU of MSH from November 2011 to July 2015 and had a TNE consultation. MSH is a highrisk fetal-maternal center and houses one of the largest tertiary perinatal centers in Canada with $~ 9000$ deliveries per annum. Its NICU admits $\sim 1000$ neonates per year, the majority of whom are inborn. Similar to many perinatal centers, MSH does not have in-house pediatric subspeciality services, including cardiology. Prior to the TNE program, the regional pediatric echocardiography laboratory, available only during office hours, exclusively provided echocardiography service. The TNE program, established in 2011, was based on a hemodynamic consultative model with attending clinicians being responsible for placing the requests for consultation on an as needed basis. A typical consult comprised of a focussed clinical assessment, a comprehensive functional echocardiogram, including hemodynamic measurements acquired using a standardized imaging protocol and provision of a clinical recommendation based on the interpretation and integration of the acquired information. The TNE imaging protocol included a structural assessment to rule out critical structural heart defects. All scans were performed by or under the supervision of a neonatologist with advanced training and expertise in TNEs. Clinical management of the patient and adherence to recommendations remained at the discretion of the attending neonatologist. Follow-up TNEs were performed based on the findings of the initial scan and patients clinical condition.

Clinical suspicion of structural heart defects other than patent foramen ovale (PFO) and PDA was an indication for a pediatric cardiology consultation and echocardiogram rather than a TNE. As per our locally agreed guidelines, each neonate who had a TNE was required to have a fullstructural scan reported by the regional pediatric cardiology service at some point during the NICU stay. This was to rule out any incidental structural defects that may not have been identified on TNE and in compliance with the ASE guidelines.

The TNE program in our unit comprises of a TNE-staff neonatologist, who is the director of the program and is responsible for reviewing and finalizing all TNE reports and one to three neonatal fellows at various stages of their TNE training. With regards to obtaining consultations, the attending neonatologist when has a hemodynamic concern for a neonate under his/her care, places a request for a TNE consultation by our unit's formalized requisition process or by contacting one of the attending TNE team members in cases of urgent requests. All TNE consult requests are then completed, reports are finalized, and findings and recommendations are communicated to the attending clinician on the same day.

\section{Patient selection}

Eligible neonates were identified from TNE reports that were archived in the echocardiography database at the Department of Pediatrics at MSH. During the first year of the program, scanned copies of the hand-written reports were stored in the database, of which illegible reports were excluded. During subsequent years, we were able to include all infants, as a copy of the computer-generated reports was available for review.

\section{Data collection}

Data collected for this study included demographic characteristics at the time of birth (gestational age, birth weight, and gender), at the time of scan (postnatal age at scan, type 
of ventilator support, oxygen requirement, and inotropic support), primary indication for scan (as defined below), and the key findings on the TNEs. We categorized the primary indications for TNE consult into one of the following:

1. Evaluation of PDA: included assessment of ductal size and appraisal of shunt volume using surrogate measures.

2. Evaluation of pulmonary hemodynamics (PH): included assessment for acute or chronic $\mathrm{PH}$, pulmonary blood flow and right ventricular function.

3. Evaluation of $\mathrm{SH}$ : included assessment of left ventricular function and output and qualitative assessment of chamber size (i.e., intravascular volume status).

4. Others (included assessment for catheter tip position for central venous lines, pericardial effusion).

Incidental structural heart defects detected during TNE were also recorded. Subsequently, the clinical management of included neonates following the consult was reviewed from health charts in order to ascertain the clinical compliance with TNE consults. This was categorized as "impact on clinical management" when changes occurred in compliance within $6 \mathrm{~h}$ of the consult or "no impact on clinical management" when TNE consult either did not recommend any change in management or when the recommended changes were not followed by the clinical team within next $6 \mathrm{~h}$. Further, the regional pediatric cardiology echocardiography reports database was also reviewed to identify reports of included neonates signed off by a pediatric cardiologist and findings with regards to structural defects aside from PDA/PFO. For the purpose of analysis, each scan of an individual patient was recorded as a separate episode.

\section{Statistical considerations}

Patient characteristics, as well as TNE-related data were evaluated using descriptive statistics. The study period was divided into 2-year epochs (2012-2013 and 2014-2015), and the average number of TNEs performed per month, as well as the distribution of indications were compared between epochs. This was done to describe longitudinal trend in resource utilization and evolution of TNE service. The proportion of TNEs that resulted in a change of management was also examined in relation to the "scan indication" and "age at scan" (categorized as $\leq 1$ week, $>1$, and $\leq 4$ weeks, and $>4$ weeks of age). Univariate analysis was performed comparing the scans that made an impact on clinical management vs. those that did not. Continuous variables were compared using student's $T$-test or Mann-Whitney $U$-test and categorical variables using chisquare test. To identify factors predictive of the impact on
Table 1 Baseline characteristics of the study group

\begin{tabular}{ll}
\hline Gestational age (week) & $26(25,28)$ (Range: 23-42) \\
Birth weight (gram) & $830(690,1040)$ \\
& $($ Range: 430-5880) \\
Number of scans per infant & $1(1,3)($ Range: 1-11) \\
Male sex & $156(59 \%)$ \\
Postnatal age at first scan (days) $^{\mathrm{a}}$ & $16(5,34)$ \\
Mechanical ventilation $^{\mathrm{a}}$ & $247(45 \%)$ \\
Receiving inotropes $^{\mathrm{a}}$ & $42(8 \%)$ \\
Indications for TNE $^{\mathrm{a}}$ & \\
PDA & $337(61 \%)$ \\
PH & $148(27 \%)$ \\
SH & $52(9 \%)$ \\
Others & $16(3 \%)$ \\
Timings of scan & \\
$\leq 1$ week & $183(33 \%)$ \\
2-4 weeks & $205(38 \%)$ \\
$>4$ weeks & $165(30 \%)$ \\
\hline
\end{tabular}

Values are median (IQR) or $n(\%)$

$\mathrm{PH}$ pulmonary hypertension, $\mathrm{SH}$ systemic hypotension

${ }^{\mathrm{a}}$ Denominator represents the total number of scans $(n=553)$

clinical management of TNEs, multivariable logistic regression analysis was performed using the first scan for each patient, including the following covariates: gestational age, birth weight, sex, postnatal age at scan, oxygen requirement, and type of ventilation at the time of scan, scan indication and epoch (2012-2013 vs. 2014-2015). Scans performed in 2011 ( 2 months) were not included in this analysis. A $p$-value $<0.05$ was considered significant.

\section{Results}

Of the total 599 scans performed during the study period, 46 were excluded due to incomplete echocardiography reports. The remaining 553 TNEs performed on 268 neonates were included in the study. Baseline characteristics of the cohort and the description of the included TNEs are shown in Table 1.

The average number of TNEs requested per month increased from nine per month during the first epoch to 20 per month during the second epoch. This was mainly due to an increase in the number of TNEs requested for non-PDA indications during the second epoch; resulting in a significant change in the proportions of scan indications over the study period (Fig. 1).

\section{Impact of TNE on clinical management}

Overall, 267 (48\%) TNEs were followed by a change in clinical management within $6 \mathrm{~h}$, which in all but two cases 


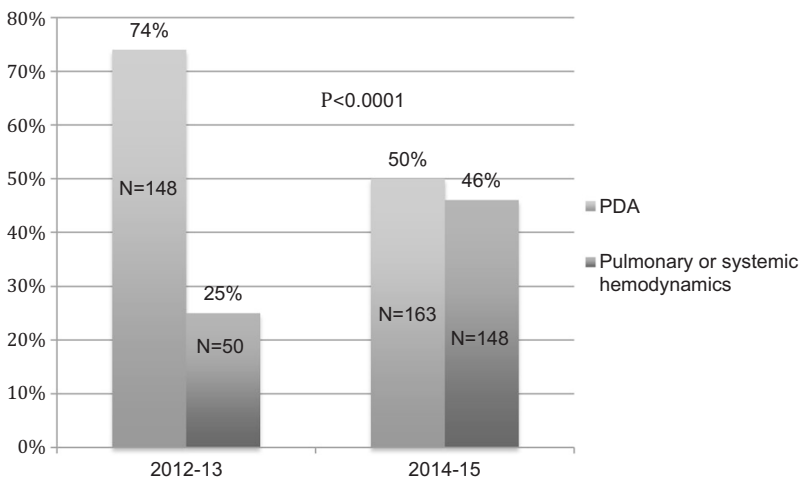

Fig. 1 Change in the indications for TNE between 2012-2013 and 2014-2015

were in compliance with the consult recommendations (99.2\%). Ninety-five consultations (17\%) made no specific recommendation and the remaining 191 (35\%) recommended continuing the same management. Impact of the TNE service also varied significantly based on the indication for the scan (Fig. 2) and to a lesser extent based on the postnatal age at scan (Fig. 3).

Comparison with scans that were associated with a change vs. no change in clinical management revealed some important differences suggestive of scans performed at the time of higher illness severity to be more likely to result in a change in management (Table 2).

Logistic regression identified male sex (adjusted odds ratio (95\% confidence interval): $1.9(1.0,3.5) ; p=0.04)$, invasive ventilation $(2.43(1.2,4.9) ; p=0.01)$, and scans assessing PH $(7.1(2.2,23.2) ; p<0.01)$ and SH (2.6 (1.1, $6.5) ; p=0.03)$ as independent predictors of TNEs resulting in a change in clinical management.

\section{TNE performed as the initial scan and unexpected findings}

Evaluation of neonates who had TNE as the initial scan before undergoing a scan performed by cardiology service $(n=81)$, revealed that TNEs successfully identified incidental structural heart defects in $10(3.7 \%)$ cases (six ventricular septal defects, three pulmonary valve stenosis, and one dysplastic mitral valve with severe mitral regurgitation), but missed identifying four cardiac defects (one mild left pulmonary artery stenosis, one persistent left superior vena cava, one small ventricular septal defect, and one left coronary artery to main pulmonary artery fistula). Overall, for $106(40 \%)$ neonates who had a TNE during NICU stay, a cardiology report could not be found in the database. There was no documentation of any cardiovascular symptoms or signs on discharge examination in any of these infants. Five TNEs were performed for sudden cardiac arrest in previously stable NICU patients; cardiac tamponade

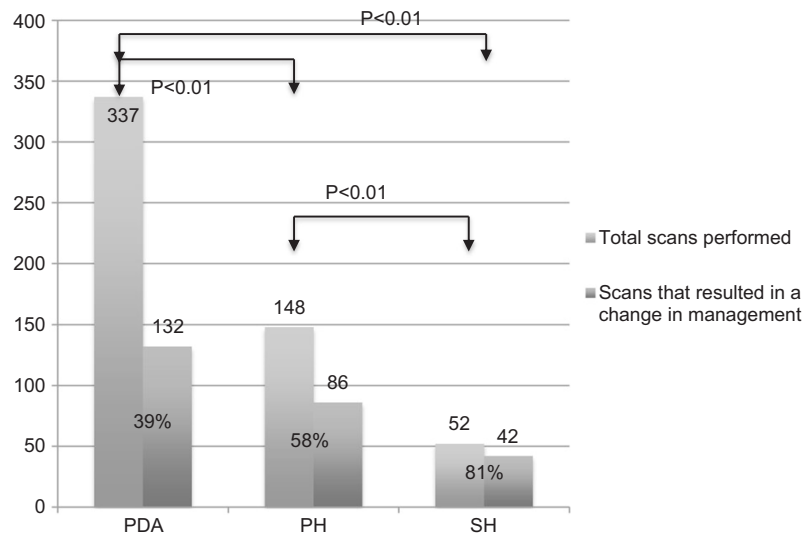

Fig. 2 Scans resulting in a change in clinical management categorized by indication

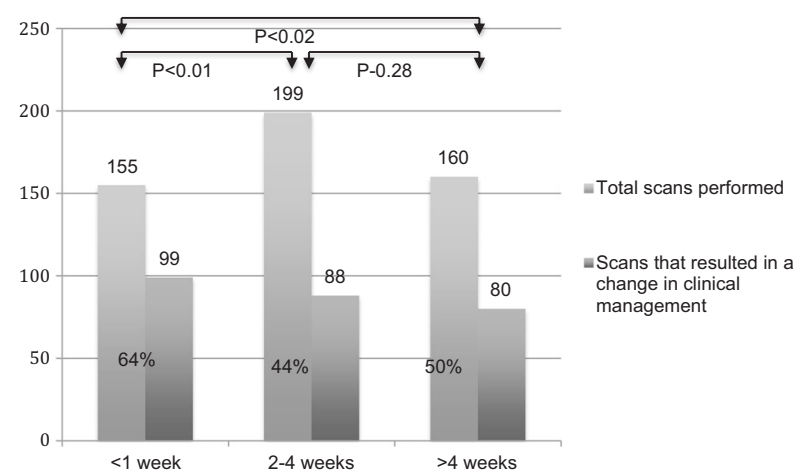

Fig. 3 Age distribution of scans that resulted in a change in clinical management

physiology secondary to TPN extravasation from central venous lines was identified in all of them for which emergency pericardiocentesis was done that proved lifesaving. Other unexpected findings identified on TNEs included 12 intra-cardiac tip positions of central venous catheters, two cases of pleural effusions, and one case of ascites.

\section{Discussion}

Although, neonatologist performed functional echocardiography has been in use in tertiary NICUs of Australia, New Zealand, and parts of Europe for a long time, its utilization in North America is relatively recent, with the vast majority of programs being established in the last decade, possible due in part to the endorsement of this modality by ASE and publication of detailed practice guidelines specific for the North American health care environment [5, 7, 10, 11]. At $\mathrm{MSH}$, a TNE and neonatal hemodynamic consult service were established after, and in compliance with, the publication of the ASE guidelines. This study was undertaken to describe the evolution of this program during the first 4 years of its inception. 
Table 2 Univariate analysis between TNEs that resulted in change in management vs. no change

\begin{tabular}{|c|c|c|c|}
\hline Characteristics & $\begin{array}{l}\text { Impact on clinical } \\
\text { management }(N=267)\end{array}$ & $\begin{array}{l}\text { No impact on clinical } \\
\text { management }(N=286)\end{array}$ & $p$-value \\
\hline Gestational age $^{\mathrm{a}}$ (weeks) & $27.4 \pm 3.9$ & $27.3 \pm 3.5$ & NS \\
\hline Birth weight (gram) ${ }^{\mathrm{a}}$ & $1019 \pm 696$ & $1049 \pm 636$ & NS \\
\hline Males $^{\mathrm{a}}$ & $69(44)$ & $87(56)$ & NS \\
\hline $\begin{array}{l}\text { Postnatal age at the time of } \\
\text { scan (days) }\end{array}$ & $16(3,34)$ & $15(7,32)$ & NS \\
\hline \multicolumn{4}{|l|}{ Main indications } \\
\hline PDA & $132(39)$ & $205(61)$ & \multirow[t]{3}{*}{$<0.01$} \\
\hline $\mathrm{PH}$ & $86(58)$ & $62(42)$ & \\
\hline $\mathrm{SH}$ & $42(81)$ & $10(19)$ & \\
\hline Mechanical ventilation & $136(55)$ & $111(45)$ & $<0.01$ \\
\hline $\mathrm{FiO}_{2}$ at the time of scan (\%) & $30(25,45)$ & $28(21,35)$ & $<0.01$ \\
\hline Receiving inotropes & $36(86 \%)$ & $6(14 \%)$ & $<0.01$ \\
\hline \multicolumn{4}{|l|}{ Scans during } \\
\hline 2012-2013 & $102(51)$ & 97 (49) & \multirow[t]{2}{*}{ NS } \\
\hline 2014-2015 & 147 (46) & $176(54)$ & \\
\hline
\end{tabular}

Values are mean $\pm \mathrm{SD}, n(\%)$ or median (IQR). $\mathrm{FiO}_{2}$-inspired oxygen concentration $P D A$ patent ductus arteriosus, $P H$ pulmonary hypertension, $S H$ systemic hypotension ${ }^{\mathrm{a}}$ Denominator is the total number of neonates $(n=268)$
A few investigators have previously described the use of neonatologist performed functional echocardiography in tertiary NICUs $[1,8,9,12,13]$. The common conclusions among these reports, as also confirmed by us in this study, are: (1) functional echocardiography, when available, is frequently utilized by neonatologists in clinical practice, (2) assessment of PDA is the most common indication for its use, with $\mathrm{PH}$ and $\mathrm{SH}$ being significant others, and (3) overall, more than one-third of TNEs result in a change in clinical management. There were some additional key findings of our study that help us further our understanding of the optimal utilization and impact on clinical management of this assessment tool.

Interestingly, we found that the indications for which clinicians requested a TNE consult evolved over time in our NICU. While PDA remained the most common reason, with the number of PDA scans remaining unchanged over time, there was a significant increase in the use of TNE consults for assessment of $\mathrm{PH} / \mathrm{SH}$ during the second half of the study period. This increase may be reflective of "consumer satisfaction," where, based on their past experiences, clinicians developed confidence in the utility of this service in patient care as it provided them with more detailed hemodynamic information such that they increasingly used it for more complex and high-acuity cases. The other plausible reasons for increased utilization of TNE service include the maturation and improved organization of the TNE consult service over time and the establishment of a formal training program at our center ensuring availability of fellows at various stages of their training. This is supported by our finding of a high percentage of $\mathrm{PH} / \mathrm{SH}$ consults resulting in a change in clinical management and that TNE consult recommendations were adopted in almost all cases by the attending clinicians. Having a large sample size and including more detailed demographic and clinical data, allowed us to examine and identify previously unreported factors associated with the impact on clinical management of TNEs. These included male sex, need for invasive ventilation and scans being requested for $\mathrm{PH}$ and $\mathrm{SH}$; all consistent with higher illness severity. Similar to Harabor et al. [9], we also noted a significantly higher percentage of TNEs performed in the first week of age resulting in changes in clinical management; albeit, a relatively lower absolute difference (50 vs. $22 \%$ in the study by Harabor et al. [9] vs. 64 vs. $47 \%$ in our study). We found, however, that this relationship between postnatal age and impact on clinical management of TNE was no longer significant after adjusting for other covariates. It was interesting to note that in nearly one-third of the consults, TNE suggested continuing the same course of action already pursued by the clinical team, which could suggest an overuse of resources vs. the clinical impact by confirmation of clinical impression. This may be valuable, and on occasions may even prevent subsequent changes in management.

The ASE training and practice guidelines for TNE, established in collaboration with European Association of Echocardiography and Association for European Pediatric Cardiologists, specifically addresses the issue of first time scans in critically ill neonates when structural heart defect is not clinically suspected [5]. It recommends that such scans 
must be comprehensive, may be performed by a core TNE person and reviewed by an advanced TNE practitioner, but should be reviewed by a pediatric cardiologist with in a "reasonable time frame," which was not defined. Although, the premise for this recommendation is to avoid potential clinical and medicolegal complications of missed diagnosis of an incidental structural heart defect, its feasibility in perinatal centers without in-house pediatric cardiologists available to review TNEs within a reasonable time frame needs evaluation. Further, performing a full-structural imaging protocol necessitates a longer scanning time; in our experience up to 45-60 min even in the hands of experts. Such handling may not be well tolerated by sick neonates and is even less desirable in preterm neonates during early postnatal age. To overcome these barriers, in collaboration with the pediatric cardiology division, we developed local guidelines suitable to our setting. First, we established a standard comprehensive TNE imaging protocol which, in experienced hands, could be performed in 15-20 min and included images to rule out incidental findings of critical congenital heart defects, especially those related to inflow and outflow tracts, cardiac chambers and valves, aortic arch and large atrial or ventricular septal defects. This TNE imaging protocol approved by our pediatric cardiology division is quicker to perform and hence more feasible in sick neonates although it does leave the possibility of not detecting minor incidental lesions. To prevent missing any congenital heart defects, all neonates without clinical suspicion of structural lesions who underwent a TNE were to electively have a full-structural scan performed and reported by a pediatric cardiologist before NICU discharge; the timing of this scan was left to the discretion of the attending clinician.

The effectiveness of our imaging protocol is confirmed by the fact that all neonates with major cardiac defect were systematically identified during the study period. The compliance with the other component of our local arrangement was rather poor, with almost $40 \%$ of TNE scans not being followed by a cardiology review. One explanation for this noncompliance could be the dependency on multiple sequential handovers between attending clinicians, especially in context of absence of clinical suspicion, as suggested by documented normal cardiac examination. It is important to acknowledge that structural heart defects may be missed on a modified TNE protocol therefore reenforcing the need for timely review of a fullstructural scan by a pediatric cardiologist. Although, the "missed" lesions were minor and none required active medical management, failure to identify these defects may have future medicolegal implications.

In addition to the common drawbacks associated with a retrospective study design, our study presents some specific limitations. Like previous similar studies, we are unable to confirm that all the changes observed after TNE consults were indeed an impact of the service. We did not record the clinical team's intended management plan prior to TNE consultation to ascertain whether recommendations led to an unanticipated change in patient management versus endorsement of the original treatment plan. Similarly, although we demonstrated an immediate impact on clinical practice, the relevance of these change to neonatal outcomes remain uncertain and requires prospective evaluation in future studies. Further, TNE requests and its indication could have been influenced by the personal biases and past experiences of attending neonatologists. We did not evaluate its impact on service utilization. Finally, a significant proportion of neonates who underwent TNE did not have a cardiology review, limiting our ability to comment on the effectiveness of this service in detecting incidental structural heart defects. Nevertheless, comparison of 81 TNEs to subsequently performed cardiology scans is still the largest such evaluation reported to date.

\section{Conclusions}

We report increasing utilization over time of an established TNE consult service in a tertiary perinatal NICU without availability of in-house cardiology services and confirm its impact on clinical service, particularly in the management of complex high-acuity cases. Male sex, treatment with invasive ventilation and $\mathrm{PH}$ or $\mathrm{SH}$ were independent predictors of TNE resulting in a change in clinical management. A standardized comprehensive TNE imaging protocol, in experienced hands and reviewed by advanced TNE personnel, may be able to identify incidental findings of major cardiac lesions, but can miss minor lesions, which may have future implications. The impact of incorporating TNE consults and related changes into clinical decision making on eventual patient outcomes needs further investigation.

\section{Compliance with ethical standards}

Conflict of interest The authors declare that they have no conflict of interest.

\section{References}

1. Sehgal A, McNamara PJ. Does point-of-care functional echocardiography enhance cardiovascular care in the NICU? J Perinatol. 2008;28:729-35.

2. El-Khuffash AF, McNamara PJ. Neonatologist-performed functional echocardiography in the neonatal intensive care unit. Semin Fetal Neonatal Med. 2011;16:50-60.

3. Jain A, McNamara PJ, Ng E, El-Khuffash A. The use of targeted neonatal echocardiography to confirm placement of peripherally 
inserted central catheters in neonates. Am $\mathrm{J}$ Perinatol. 2012;29:101-6.

4. Mukerji A, Diambomba Y, Lee SK, Jain A. Use of targeted neonatal echocardiography and focused cardiac sonography in tertiary neonatal intensive care units: time to embrace it? J Ultrasound Med. 2016;35:1579-91.

5. Mertens L, Seri I, Marek J, Arlettaz R, Barker P, McNamara P, et al. Targeted neonatal echocardiography in the neonatal intensive care unit: practice guidelines and recommendations for training. Writing group of the American Society of Echocardiography (ASE) in collaboration with the European Association of Echocardiography (EAE) and the Association for European Pediatric Cardiologists (AEPC). J Am Soc Echocardiogr. 2011;24:1057-78.

6. Singh Y, Gupta S, Groves AM, Gandhi A, Thomson J, Qureshi S, et al. Expert consensus statement 'Neonatologist-performed Echocardiography (NoPE)'-training and accreditation in UK. Eur J Pediatr. 2016;175:281-7.

7. Mertens L, Seri I, Marek J, Arlettaz R, Barker P, McNamara P, et al. Targeted neonatal echocardiography in the neonatal intensive care unit: practice guidelines and recommendations for training. Eur J Echocardiogr. 2011;12:715-36.
8. El-Khuffash A, Herbozo C, Jain A, Lapointe A, McNamara PJ. Targeted neonatal echocardiography (TnECHO) service in a Canadian neonatal intensive care unit: a 4-year experience. J Perinatol. 2013;33:687-90.

9. Harabor A, Soraisham AS. Utility of targeted neonatal echocardiography in the management of neonatal illness. J Ultrasound Med. 2015;34:1259-63.

10. Finan E, Sehgal A, Khuffash AE, McNamara PJ. Targeted neonatal echocardiography services: need for standardized training and quality assurance. J Ultrasound Med. 2014;33:1833-41.

11. Evans N. Echocardiography on neonatal intensive care units in Australia and New Zealand. J Paediatr Child Health. 2000;36:169-71.

12. Corredera A, Rodriguez MJ, Arevalo P, Llorente B, Moro M, Arruza L. [Functional echocardiography in neonatal intensive care: 1 year experience in a unit in Spain]. An Pediatr. 2014;81:167-73.

13. Moss S, Kitchiner DJ, Yoxall CW, Subhedar NV. Evaluation of echocardiography on the neonatal unit. Arch Dis Child Fetal Neonatal Ed. 2003;88:F287-9. discussionF90-1. 